\title{
Event-triggered Consensus of Heterogeneous Multi-agent Systems with Double-Integrator Dynamics
}

\author{
Dong Xue and Sandra Hirche
}

\begin{abstract}
This paper studies the second-order consensus problem for multi-agent systems (MASs) associated with an event-triggered update policy. We first investigate the topological properties of the heterogeneous position and velocity information graph to guarantee consensus. Then, consensus protocols using an event-triggered update law are analyzed for the centralized and distributed cases. In particular, we establish an event-triggered scheduling policy ensuring that the MAS exponentially achieves consensus. Furthermore, a positive lower bound for inter-event times is derived to guarantee the absence of Zeno behavior. Finally, simulations are provided to demonstrate the effectiveness of proposed approach.
\end{abstract}

\section{INTRODUCTION}

Consensus problems of MASs have become an active area of research [1] due to the advances in information, communication and computation technologies. In the related literature agents are often considered to be governed by first-order or single-integrator dynamics [1]. Meanwhile, second-order dynamics have received growing attention as many MASs are characterized by two states per agent, often position and velocity [2], [3]. It is well known that the consensus problem with double-integrator dynamics is more challenging than that with single-integrator dynamics.

Most works on second-order consensus assume that the information, such as position and velocity, is exchanged along a homogeneous communication network, i.e. the underlying graph topologies for the position and velocity information exchange are identical. However, sometimes measurements of position and velocity are not available for each single agent due to higher cost of velocity sensors. Only few works have investigated the potential advantages and drawbacks of different information topologies for different measurement signals, e.g. [3]. In this paper we investigate topological properties to guarantee the heterogeneous double integrator network achieving consensus.

In practice, a MAS achieves consensus by exchanging information over a communication network, very often wireless communication is considered. The communication is subject to limited communication bandwidth or limited battery capacity of intermediate transmission nodes in wireless networks. Thus, the efficient utilization of such communication resources is one of the important challenges in networked MASs. The scheduling of the control updates meeting a trade-off between control performance and number of update transmissions can be implemented in a time-

D. Xue and S. Hirche are with the Institute for Information-oriented Control, Technische Universität München, Arcisstraße 21, D-80290 München, Germany; dong.xuedtum.de, hirchedtum.de. or event-driven method. The former approach typically involves periodic sampling, which leads to significant overprovisioning of network resources since the predetermined task period is determined by a worst situation time interval in order to assure the system performance. In the past years, aperiodic schemes have been proposed, in which the information transmission is executed in an event-triggered fashion [4]-[6]. In event-triggering schemes the system state is sampled/transmitted when a certain internal measurement function exceeds a threshold. Advantageously, the eventdriven control improves the overall control system performance while maintaining the utilization rate of the communication resources, see e.g. [4].

The design of the event-triggering rule and the control law aims at preserving the desired properties of control systems, such as stability [5] and convergence [7]. In [5], an eventtriggered strategy is developed to schedule the control tasks preserving input-to-state stability (ISS) with respect to the measurement errors. A so-called $\mathcal{L}_{2}$ event-trigger is proposed in [6] which preserves the induced $\mathcal{L}_{2}$ gain in the closedloop system. Regarding the fact that consensus algorithms are input-to-state stable in [8], the event-triggered protocols with respect to ISS are introduced into cooperative control of single-integrator MAS [7]. To the best of our knowledge, there is little prior work on event-triggered control for MASs with double-integrator dynamics, e.g. [9].

The main contribution of this paper is an event-triggered control law to guarantee the second-order consensus for MASs with heterogeneous information topologies. We show that the proposed event-triggered policy guarantees consensus. Moreover, both cases of centralized and distributed event-triggered consensus control are considered in this paper. In the context of distributed event-triggered control, each agent independently updates its own control input based on the local information. We show the existence of a lower bound on the time between consecutive executions of the actuation update and guarantee the absence of Zeno behavior. Numerical examples successfully show the validity of the proposed approach.

The remainder of the paper is organized as follows: In Section II, some necessary background and the model formulation are given; the main results are presented in Section III; numerical examples are given in Section IV.

Notation 1.1: Throughout this paper, $\mathbb{N}, \mathbb{R}$ and $\mathbb{C}$ denote the set of non-negative integers, real numbers and complex numbers, respectively. $\operatorname{det}(\cdot)$ denotes the determinant of a matrix and $\operatorname{diag}(\cdot)$ denotes the diagonal matrix. 1 and $\mathbf{0}$ represent the column vector of all ones and all zeros 
with appropriate dimensions, respectively. $I^{n}\left(\mathbf{0}^{n}\right)$ is the ndimensional identity (zero) matrix. The conjugate transpose of the matrix $A$ is written $A^{\dagger} . \operatorname{Re}(\mu)$ is the real part of a complex $\mu .\|\cdot\|$ denotes the Euclidean norm for vectors and the induced 2-norm for matrices.

\section{BACKGROUND AND PROBLEM STATEMENT}

In this section, some background of graph and matrix theory are first presented followed by the model formulation and problem statement.

\section{A. Algebraic Graph Theory}

The following results and further details on graph theory are found in [10]. Let $\mathcal{G}=(\mathcal{V}, \mathcal{E})$ be a graph with $n$ vertices, where $\mathcal{V}=\left\{\mathrm{v}_{1}, \ldots, \mathrm{v}_{n}\right\}$ and $\mathcal{E} \subseteq \mathcal{V} \times \mathcal{V}$ are the set of vertices and edges, respectively. The set of neighbors of node $i$ is denoted by $\mathcal{N}_{i}=\left\{\mathrm{v}_{j} \in \mathcal{V}:\left(\mathrm{v}_{i}, \mathrm{v}_{j}\right) \in \mathcal{E}\right\}$. The adjacency matrix $A(\mathcal{G})=\left(a_{i j}\right)_{n \times n}$ of an undirected graph is defined such that $a_{i j}=a_{j i}=1$ if there is a connection between vertex $i$ and $j(j \neq i)$. The degree of a node is given by $d\left(\mathrm{v}_{i}\right)=\sum_{j} a_{i j}$. Let the Laplacian matrix $L(\mathcal{G})=\left[l_{i j}\right] \in \mathbb{R}^{n \times n}$ associated with $A(\mathcal{G})$ be defined as $L(\mathcal{G})=\operatorname{diag}\left(d\left(\mathrm{v}_{i}\right)\right)-A(\mathcal{G})$ (for simplicity of notation, $L$ is used in the following paper).

In this paper, undirected graphs are considered. For an undirected graph, $L$ is symmetric positive semi-definite and hence there exist $n$ linearly independent eigenvectors. Furthermore, all nonzero eigenvalues of $L$ are positive and real. 0 is an eigenvalue of $L$ with the associated right (left) eigenvector $\mathbf{1}\left(\mathbf{1}^{\top}\right)$, i.e., $L \mathbf{1}=\mathbf{0}$. A graph is connected if and only if 0 is simple eigenvalue of the Laplacian matrix.

\section{B. System Model}

Consider $n$ agents with double-integrator dynamics

$$
\dot{x}_{i}(t)=v_{i}(t), \quad \dot{v}_{i}(t)=u_{i}(t), \quad i \in\{1, \ldots, n\},
$$

where $x_{i} \in \mathbb{R}^{m}$ and $v_{i} \in \mathbb{R}^{m}$ are position and velocity vectors of agent $i$ with initial states $x_{i}\left(t_{0}\right)=x_{i}(0)$ and $v_{i}\left(t_{0}\right)=v_{i}(0)\left(t_{0}\right.$ is the initial time $) ; u_{i} \in \mathbb{R}^{m}$ is the control input to be designed based on the information received by agent $i$. Note that $x_{i}$ and $v_{i}$ can also represent other physical quantities. To facilitate the following analysis, the one-dimensional case is considered in this paper, i.e. $m=1$. Analogous results for higher dimensional systems are obtained by means of the Kronecker product.

Let $\mathcal{G}_{x}$ and $\mathcal{G}_{v}$ be the information graphs with respect to positions and velocities, respectively. The corresponding Laplacian matrices are defined as $L_{x}$ and $L_{v}$. In this paper, we consider the general case where the information graphs for position and velocity exchange might be different, i.e. $L_{x} \neq L_{v}$. It is worth mentioning that the second-order consensus discussed in [2], where $L_{v}=L_{x}$ can be recovered as a special case of this paper. Since the graphs $\mathcal{G}_{x}=\left(\mathcal{V}, \mathcal{E}_{x}\right)$ and $\mathcal{G}_{v}=\left(\mathcal{V}, \mathcal{E}_{v}\right)$ share the same set of nodes, any operations (such as adding, removing and renaming nodes) are executed accordingly. For each agent $i$, two subsets $\mathcal{N}_{i}\left(\mathcal{G}_{x}\right) \subset \mathcal{V}$ and $\mathcal{N}_{i}\left(\mathcal{G}_{v}\right) \subset \mathcal{V}$ are given to identify the agents with which it can exchange position and velocity information. $\mathcal{N}_{i}\left(\mathcal{G}_{x}\right)$ and $\mathcal{N}_{i}\left(\mathcal{G}_{v}\right)$ are replaced by $\mathcal{N}_{i}^{x}$ and $\mathcal{N}_{i}^{v}$ for convenience in the following analysis.

The consensus algorithm for (1) is proposed as

$$
u_{i}=-\alpha \sum_{j \in \mathcal{N}_{i}^{x}}\left(x_{i}-x_{j}\right)-\beta \sum_{j \in \mathcal{N}_{i}^{v}}\left(v_{i}-v_{j}\right) .
$$

where $\alpha>0$ and $\beta>0$ are the respective coupling strengths.

Let $x=\left[x_{1}^{\top}, \ldots, x_{n}^{\top}\right]^{\top}, v=\left[v_{1}^{\top}, \ldots, v_{n}^{\top}\right]^{\top}$, then applying the control law (2), the closed loop dynamics of the MAS (1) can be rewritten in a compact matrix form as

$$
\left[\begin{array}{c}
\dot{x} \\
\dot{v}
\end{array}\right]=\underbrace{\left[\begin{array}{cc}
0^{n} & I^{n} \\
-\alpha L_{x} & -\beta L_{v}
\end{array}\right]}_{\mathcal{L}}\left[\begin{array}{l}
x \\
v
\end{array}\right],
$$

where $\mathcal{L}$ represents the overall system matrix.

Definition 2.1: Consider the MAS (3), the secondorder consensus is said to be achieved if for any initial condition, one has $\lim _{t \rightarrow \infty}\left(x_{i}(t)-x_{j}(t)\right)=0$, and $\lim _{t \rightarrow \infty}\left(v_{i}(t)-v_{j}(t)\right)=0, \forall i, j \in\{1, \ldots, n\}$.

\section{Main Results}

In this section, we will investigate under which condition on the graph topologies $\mathcal{G}_{x}$ and $\mathcal{G}_{v}$ second-order consensus is achieved via the analysis of the spectrum of the matrix $\mathcal{L}$. Furthermore, the convergence of the consensus protocols associated with event-triggered update policy is investigated.

\section{A. Spectrum Analysis of Matrix $\mathcal{L}$}

In the second-order model (3), the eigenvalues of the matrix $\mathcal{L}$ play an important role for the convergence of the system. It is worthy to note that, as a consequence of the asymmetry of matrix $\mathcal{L}$, one should expect to have complex entries in its eigenvectors.

For a nonzero vector $\omega$, the Rayleigh quotient of $\omega$ w.r.t. the real symmetric matrix $L_{x}$ and $L_{v}$ are defined as

$$
\mathcal{R}\left(L_{x}, \omega\right)=\frac{\omega^{\dagger} L_{x} \omega}{\omega^{\dagger} \omega} \geq 0, \quad \mathcal{R}\left(L_{v}, \omega\right)=\frac{\omega^{\dagger} L_{v} \omega}{\omega^{\dagger} \omega} \geq 0,
$$

where the nonnegativity is derived from the positive semidefiniteness of Laplacian matrix of undirected graph. As in this paper, the Rayleigh quotient is associated with the Laplacian matrix, and for simplicity of notation we write $\mathcal{R}_{x}(\omega)$ instead of $\mathcal{R}\left(L_{x}, \omega\right)$.

Lemma 3.1: All the eigenvalues of $\mathcal{L}$ lie in the closed left half plane of $\mathbb{C}$.

Proof: The characteristic polynomial of matrix $\mathcal{L}$ is

$$
P(\lambda)=\operatorname{det}\left(\lambda I^{2 n}-\mathcal{L}\right)=\operatorname{det}(\underbrace{\lambda^{2} I^{n}+\beta \lambda L_{v}+\alpha L_{x}}_{:=Q(\lambda)}),
$$

where the $\lambda$-matrix $Q(\lambda)$ is an $n \times n$ matrix polynomial of degree 2. It is shown in [11] that $Q(\lambda)$ has the same spectrum as $\mathcal{L}$ and we can investigate the spectrum of $Q(\lambda)$ instead of $\mathcal{L}$. Due to the fact that Laplacian matrices are real, all the eigenvalues of $Q(\lambda)$ are symmetric with respect to the real axis of the complex plane. Let $(\lambda, \omega)$ be an eigenpair of $Q(\lambda)$, i.e. $Q(\lambda) \omega=0$, which leads to $\omega^{\dagger} Q(\lambda) \omega=0$. 
Therefore, an explicit formula for the eigenvalues of matrix polynomial $Q(\lambda)$ can be given as

$$
\lambda_{ \pm}=-\frac{\beta \mathcal{R}_{v}(\omega)}{2} \pm \sqrt{\frac{\beta^{2} \mathcal{R}_{v}^{2}(\omega)}{4}-\alpha \mathcal{R}_{x}(\omega)} .
$$

From above formulae, it is straightforward to show that all the eigenvalues of $\mathcal{L}$ have a nonpositive real part.

Lemma 3.2: [2] The MAS in (3) achieves second-order consensus asymptotically if and only if matrix $\mathcal{L}$ has exactly one zero eigenvalue of algebraic multiplicity 2 and geometric multiplicity 1 , and all the other nonzero eigenvalues have negative real parts.

Note that for a nonzero vector $\omega$, if $(\lambda, \omega)$ is an eigenpair of $Q(\lambda)$, then the vector $\xi=\left[\begin{array}{c}\omega \\ \lambda \omega\end{array}\right]$ is an eigenvector associated with eigenvalue $\lambda$ for $\mathcal{L}$. In particular, $(0,1)$ is an eigenpair of $Q(\lambda)$ and we can straightforwardly verify that $\left(0,\left[\begin{array}{l}\mathbf{1} \\ \mathbf{0}\end{array}\right]\right)$ is an eigenvalue pair of $\mathcal{L}$. In addition, due to $\mathbf{1} \in \operatorname{ker} L_{x} \cap \operatorname{ker} L_{v}$, the Rayleigh quotients $\mathcal{R}_{x}(\mathbf{1})=\mathcal{R}_{v}(\mathbf{1})=0$. Furthermore, if $\omega$ is an eigenvector of $L_{x}$ associated with a zero eigenvalue, i.e. $\omega \in \operatorname{ker} L_{x}$, then $\mathcal{R}_{x}(\omega)=0$. In (5), when $\mathcal{R}_{x}(\omega)=0$, the $\lambda$-matrix $Q(\lambda)$ has two zero eigenvalues. The next lemma is quite obvious from previous reasoning, therefore the proof is omitted here.

Lemma 3.3: $\lambda=0$ is an eigenvalue of $\mathcal{L}$ and $\left[\begin{array}{l}\mathbf{1} \\ \mathbf{0}\end{array}\right]$ is the associated right eigenvector. Moreover, the geometric multiplicity of eigenvalue $\lambda=0$ equals the number of connected components contained in the position graph.

Lemma 3.4: The MAS (3) achieves consensus only if the position graph $\mathcal{G}_{x}$ is connected.

Proof: $\quad$ Straightforward from Lemma 3.2 and Lemma 3.3.

Next, the case of purely imaginary eigenvalues in $\mathcal{L}$ is eliminated, since it fails to guarantee the convergence of the second-order MAS. According to (5), the purely imaginary eigenvalues exist only when $R_{v}(\omega)=0$ while $R_{x}(\omega) \neq 0$.

Proposition 3.1: Consider the MAS (1) together with the control law (2). If the graph $\mathcal{G}_{x}$ and $\mathcal{G}_{v}$ are both connected, then the matrix $\mathcal{L}$ has exactly one zero eigenvalue with algebraic multiplicity 2 and geometric multiplicity 1 , and all the other nonzero eigenvalues have negative real parts.

Proof: According to Lemma 3.3, for connected position graph, the Jordan canonical form of $\mathcal{L}$ has one Jordan block corresponding to the zero eigenvalue. Furthermore, $\left[\begin{array}{l}\mathbf{1} \\ \mathbf{0}\end{array}\right]$ is one of the right eigenvector associated with zero eigenvalue and $\left[\begin{array}{l}0 \\ 1\end{array}\right]$ is a generalized eigenvector due to $\mathcal{L}\left[\begin{array}{l}0 \\ 1\end{array}\right]=\left[\begin{array}{l}\mathbf{1} \\ \mathbf{0}\end{array}\right]$. In addition, there is no vector $\left[\begin{array}{l}p_{1} \\ p_{2}\end{array}\right]$ such that $\mathcal{L}\left[\begin{array}{l}p_{1} \\ p_{2}\end{array}\right]=\left[\begin{array}{l}0 \\ 1\end{array}\right]$, which is derived from the fact that $p_{2}=\mathbf{0}$ here and there is no $p_{1}$ that satisfies $-\alpha L_{x} p_{1}=1$. Hence, matrix $\mathcal{L}$ has exactly one zero eigenvalue with algebraic/geometric multiplicity $2 / 1$. The remainder of the proof is derived by using Lemma 3.1 and the result that for the connected position and velocity graphs, a vector $\omega$ satisfies $R_{v}(\omega)=0$ which leads to $R_{x}(\omega)=0$.

In the sequel of the paper, we call the graph pair $\mathcal{G}_{x}$ and $\mathcal{G}_{v}$ heterogeneously connected if both are connected but their topologies are not necessary identical.

\section{B. Distributed Event-triggered Control}

In contrast to traditional time-triggered sampling approaches, the control law considered in this paper is updated at instants triggered by events, specifically, at times when the measurement error exceeds a certain threshold. In particular, we investigate the distributed case in the sense that each agent locally decides the event instants. By "local" we mean, that the decision on an event only depends on local information from its neighboring agents.

The sequence of event times for each agent $i \in\{1, \ldots, n\}$ is denoted by $\left\{t_{k}^{i}\right\}_{k \in \mathbb{N}}$. We assume that they are determined by an event-function $f_{i}(\cdot)$ which triggers an event at agent $i$ as soon as it crosses zero, i.e. $t_{k+1}^{i}=\inf \left\{t: t>t_{k}^{i}, f_{i}(\cdot)>0\right\}$. Furthermore, we assume that the control is piecewise constant between consecutive updates and equals the value of the last control update, i.e. having a zero-order hold characteristics. The distributed control law for agent $i$ is updated not only at its own eventtriggered time instants $\left\{t_{k}^{i}\right\}_{k \in \mathbb{N}}$ but also the last event times of its neighbors $\left\{t_{k}^{j}\right\}_{k \in \mathbb{N}}, j \in\left\{\mathcal{N}_{i}^{x} \cup \mathcal{N}_{i}^{v}\right\}$.

For each $t \in\left[t_{k}^{i}, t_{k+1}^{i}\right)$, the distributed event-triggered control law is given by

$$
\begin{aligned}
u_{i}(t)= & -\alpha \sum_{j \in \mathcal{N}_{i}^{x}}\left(x_{i}\left(t_{k}^{i}\right)-x_{j}\left(t_{k^{\prime}(t)}^{j}\right)\right)-\beta \sum_{j \in \mathcal{N}_{i}^{v}}\left(v_{i}\left(t_{k}^{i}\right)\right. \\
& \left.-v_{j}\left(t_{k^{\prime}(t)}^{j}\right)\right), \quad i \in\{1, \ldots, n\}, k \in \mathbb{N}
\end{aligned}
$$

where $k^{\prime}(t) \triangleq \arg _{l \in \mathbb{N}} \min _{t \geq t_{l}^{j}}\left\{t-t_{l}^{j}\right\}$, which is the index for the last event time of agent $j$ before the time $t$. The control law at agent $i$ is calculated using the most recently received states for the position and velocity of itself and its "neighbors".

Remark 3.1: In [9], a first-order hold scheme is introduced to estimate the position state at the controller between update times. However, due to the heterogeneous characteristics of second-order MAS in this paper, the position and velocity of each agent are not necessarily simultaneously available from its neighbors.

Let $\theta^{x}$ and $\theta^{v}$ be the vectors of measurement errors for position and velocity, and for the second-order agent $i$ they are defined as

$$
\begin{aligned}
& \theta_{i}^{x}(t)=x_{i}\left(t_{k}^{i}\right)-x_{i}(t), \\
& \theta_{i}^{v}(t)=v_{i}\left(t_{k}^{i}\right)-v_{i}(t), \quad t \in\left[t_{k}^{i}, t_{k+1}^{i}\right), i \in\{1, \ldots, n\} .
\end{aligned}
$$

Note that at the event instant they are reset to zero. Based on the definition of $k^{\prime}(t)$, it follows that $x_{j}\left(t_{k^{\prime}(t)}^{j}\right)=\theta_{j}^{x}(t)+x_{j}(t)$ and $v_{j}\left(t_{k^{\prime}(t)}^{j}\right)=\theta_{j}^{v}(t)+v_{j}(t)$. The second-order dynamics of agent $i$ under the distributed event-triggered control law (6) can be rewritten by

$$
\begin{aligned}
\dot{x}_{i}(t)= & v_{i}(t), \\
\dot{v}_{i}(t)= & -\alpha \sum_{j \in \mathcal{N}_{i}^{x}}\left(\theta_{i}^{x}(t)-\theta_{j}^{x}(t)+x_{i}(t)-x_{j}(t)\right) \\
& -\beta \sum_{j \in \mathcal{N}_{i}^{v}}\left(\theta_{i}^{v}(t)-\theta_{j}^{v}(t)+v_{i}(t)-v_{j}(t)\right),
\end{aligned}
$$


and hence, the entire MAS model becomes

$$
\left[\begin{array}{c}
\dot{x} \\
\dot{v}
\end{array}\right]=\mathcal{L}\left[\begin{array}{l}
x \\
v
\end{array}\right]+\hat{L} \theta
$$

where $\theta=\left[\left(\theta^{x}\right)^{\top},\left(\theta^{v}\right)^{\top}\right]^{\top}$ and $\hat{L}=\left[\begin{array}{cc}\mathbf{0}^{n} & \mathbf{0}^{n} \\ -\alpha L_{x} & -\beta L_{v}\end{array}\right]$.

From the Proposition 3.1, the assumption that $\mathcal{G}_{x}$ and $\mathcal{G}_{v}$ are heterogeneously connected implies $\mathcal{L}$ has exactly two zero eigenvalues which have geometric multiplicity 1 associated with partial multiplicity 2 . Furthermore, the remaining eigenvalues have strictly negative real parts. As a result, $\mathcal{L}$ can be written in the Jordan canonical form as

$$
\mathcal{L}=P J P^{-1}=\left[\begin{array}{lll}
p_{1} & \cdots & p_{2 n}
\end{array}\right]\left[\begin{array}{ccc}
0 & 1 & \mathbf{0}^{\top} \\
0 & 0 & \mathbf{0}^{\top} \\
\mathbf{0} & \mathbf{0} & \tilde{J}
\end{array}\right]\left[\begin{array}{c}
q_{1}^{\top} \\
\vdots \\
q_{2 n}^{\top}
\end{array}\right],
$$

where $p_{i} \in \mathbb{R}^{2 n}$ can be chosen to be the right eigenvectors or generalized eigenvectors of $\mathcal{L}$, and $q_{i} \in \mathbb{R}^{2 n}$ are the left eigenvectors or generalized eigenvectors of $\mathcal{L}$. Without loss of generality, let $p_{1}=\left[\mathbf{1}^{\top}, \mathbf{0}^{\top}\right]^{\top}, \quad p_{2}=\left[\mathbf{0}^{\top}, \mathbf{1}^{\top}\right]^{\top}, \quad q_{1}=\left[\eta^{\top}, \mathbf{0}^{\top}\right]^{\top}$ and $\quad q_{2}=\left[\mathbf{0}^{\top}, \eta^{\top}\right]^{\top}, \quad$ where $\quad \eta \in \operatorname{ker} L_{x} \cap \operatorname{ker} L_{v}$ and $\quad \eta^{\top} \mathbf{1}=1$. The remaining Jordan blocks $\quad \tilde{J} \in \mathbb{R}^{(2 n-2) \times(2 n-2)}$ are upper diagonal corresponding to non-zero eigenvalues.

Define $z(t)=P^{-1}\left[x(t)^{\top} v(t)^{\top}\right]^{\top}=\left[z_{1}^{\top} \ldots z_{2 n}^{\top}\right]^{\top}$ and its derivative is

$$
\dot{z}=P^{-1} \mathcal{L} P z+P^{-1} \hat{L} \theta=J z+P^{-1} \hat{L} \theta .
$$

As the first two rows of $P^{-1} \hat{L}$ are equal to zero, we have

$$
\dot{z}_{1}(t)=z_{2}(t), \quad \dot{z}_{2}(t)=0 .
$$

Proposition 3.2: Suppose that the MAS with the position graph $\mathcal{G}_{x}$ and velocity graph $\mathcal{G}_{v}$ is heterogeneously connected. Then, second-order consensus in MAS (7) is reached if $\lim _{t \rightarrow \infty}\left\|z_{i}(t)\right\|=0$ for $i \in\{3, \ldots, 2 n\}$. Specifically, $\quad x_{i}(t) \rightarrow \eta^{\top} x(0)+t \eta^{\top} v(0)$ and $v_{i}(t) \rightarrow \eta^{\top} v(0)$, as $t \rightarrow \infty$, for all $i \in\{1, \ldots, n\}$, where $\eta^{\top} L_{x}=\eta^{\top} L_{v}=0$ and $\eta^{\top} \mathbf{1}=1$.

Proof: Since the position graph $\mathcal{G}_{x}$ and velocity graph $\mathcal{G}_{v}$ are heterogeneously connected, $p_{1}=\left[\mathbf{1}^{\top}, \mathbf{0}^{\top}\right]^{\top}$ and $p_{2}=\left[\mathbf{0}^{\top}, \mathbf{1}^{\top}\right]^{\top}$ are the two right eigenvectors of matrix $\mathcal{L}$ associated with the zero eigenvalue. Consider $\lim _{t \rightarrow \infty}\left\|z_{i}(t)\right\|=0$ for $i=3, \ldots, 2 n$ and the definition of $P$, one gets

$$
\begin{aligned}
\lim _{t \rightarrow \infty}\left\|\left[\begin{array}{c}
x \\
v
\end{array}\right]-[\underbrace{z_{1}, \ldots, z_{1}}_{n}, \underbrace{z_{2}, \ldots, z_{2}}_{n}]^{\top}\right\| \\
=\lim _{t \rightarrow \infty}\left\|P z-[\underbrace{z_{1}, \ldots, z_{1}}_{n}, \underbrace{z_{2}, \ldots, z_{2}}_{n}]^{\top}\right\|=0
\end{aligned}
$$

where $z_{1}$ and $z_{2}$ are the solutions of (9). The equation (10) implies that, as time evolves, all agents achieve a common position $z_{1}$ and a common velocity $z_{2}$. By solving (9), the analytic solutions can be obtained as $z_{1}(t)=\eta^{\top} x(0)+t \eta^{\top} v(0)$ and $z_{2}(t)=\eta^{\top} v(0)$, where $\eta^{\top} L_{x}=\eta^{\top} L_{v}=0$ and $\eta^{\top} \mathbf{1}=1$.
Before moving on, the following notations are necessary for presenting the main results of this paper. Let $\left\{\lambda_{1}, \lambda_{2}, \lambda_{3}, \ldots, \lambda_{2 n}\right\}$ be an ordered sequence of eigenvalues for matrix $\mathcal{L}$, specifically, $0=\lambda_{1}=\lambda_{2} \geq \operatorname{Re}\left(\lambda_{3}\right) \geq$ $\ldots \geq \operatorname{Re}\left(\lambda_{2 n}\right)$. For presentation simplicity, let $M_{q}=\|\hat{L}\|$ and $M_{p}=\left\|P^{-1}\right\|\|P\|$ is equivalent to the quotient of largest singular value of $P$ divided by the smallest one.

Theorem 3.1: Consider the MAS (1) associated with distributed control law (6) and event times $\left\{t_{k}^{i}\right\}_{k=0}^{\infty}$ which is implicitly given by the update rule

$$
f_{i}\left(t, \theta_{i}^{x}(t), \theta_{i}^{v}(t)\right)=\left\|\begin{array}{l}
\theta_{i}^{x}(t) \\
\theta_{i}^{v}(t)
\end{array}\right\|-\sigma_{i} e^{-\gamma_{i} t}>0
$$

where $0<\sigma_{i}$ and $0<\gamma_{i}<\left|\operatorname{Re}\left(\lambda_{3}\right)\right|$ for $i \in\{1, \ldots, n\}$. If the position graph $\mathcal{G}_{x}$ and velocity graph $\mathcal{G}_{v}$ are heterogeneously connected, then consensus is achieved for any initial condition. Furthermore, there exists a lower bound on the inter-event interval $\left\{t_{k+1}^{i}-t_{k}^{i}\right\}_{k \in \mathbb{N}}$ for all agents.

Proof: According to Proposition 3.2, for $i=3, \ldots, 2 n$, if $\lim _{t \rightarrow \infty}\left\|z_{i}(t)\right\|=0$, then the MAS (7) achieves consensus asymptotically. To facilitate the following analysis, the $P^{-1}$ is decomposed as $q_{i}=\left[q_{i_{1}}^{\top}, v_{i}^{\top}\right]^{\top}$, where $q_{i_{1}} \in \mathbb{R}^{n}, v_{i}=\left[v_{i 1}, \ldots, v_{i n}\right]^{\top} \in \mathbb{R}^{n}, i \in\{3, \ldots, 2 n\}$.

For $i \in\{3, \ldots, 2 n\}$ in (8), we have

$$
\dot{z}_{i}=\lambda_{i} z_{i}-\alpha v_{i}^{\top} L_{x} \theta^{x}-\beta v_{i}^{\top} L_{v} \theta^{v},
$$

and its analytical solution can be calculated as follows:

$$
z_{i}=e^{\lambda_{i} t} z_{i}(0)-\int_{t_{0}}^{t} e^{\lambda_{i}(t-s)} v_{i}^{\top}\left(\alpha L_{x} \theta^{x}+\beta L_{v} \theta^{v}\right) d s .
$$

The norm of $z_{i}$ is bounded by

$$
\begin{gathered}
\left\|z_{i}\right\| \leq e^{\operatorname{Re}\left(\lambda_{i}\right) t}\left\|z_{i}(0)\right\|+\int_{t_{0}}^{t} e^{\operatorname{Re}\left(\lambda_{i}\right)(t-s)}\left\|v_{i}\right\| \\
\left\|-\alpha L_{x} \quad-\beta L_{v}\right\|\|\theta\| d s .
\end{gathered}
$$

By taking the event-triggered function (11) into account, the Euclidean norm of $\theta$ can be expressed as

$$
\|\theta(t)\|=\sqrt{\sum_{j=1}^{n}\left\|\begin{array}{l}
\theta_{j}^{x} \\
\theta_{j}^{v}
\end{array}\right\|^{2}} \leq \sum_{j=1}^{n} \sigma_{j} e^{-\gamma_{j} t} .
$$

With $\left\|-\alpha L_{x}-\beta L_{v}\right\|=M_{q}$, the norm in (12) is upper bounded

$$
\begin{aligned}
\left\|z_{i}\right\| & \leq e^{\operatorname{Re}\left(\lambda_{i}\right) t}\left(\left\|z_{i}(0)\right\|+M_{q}\left\|v_{i}\right\| \sum_{j=1}^{n} \sigma_{j} \int_{t_{0}}^{t} e^{-\left(\operatorname{Re}\left(\lambda_{j}\right)+\gamma_{j}\right) s} d s\right) \\
& \leq e^{\operatorname{Re}\left(\lambda_{i}\right) t}\left\|z_{i}(0)\right\|+\sum_{j=1}^{n} \frac{M_{q}\left\|v_{i}\right\| \sigma_{j}}{-\left(\operatorname{Re}\left(\lambda_{i}\right)+\gamma_{j}\right)} e^{-\gamma_{j} t} .
\end{aligned}
$$

Since $0<\gamma_{j}<\left|\operatorname{Re}\left(\lambda_{3}\right)\right| \leq\left|\operatorname{Re}\left(\lambda_{i}\right)\right|$ for $j \in\{1, \ldots, n\}$ and $i \in\{3, \ldots, n\}, \lim _{t \rightarrow \infty}\left\|z_{i}(t)\right\|=0$ can be derived from (13), which implies the consensus of MAS is reached.

In order to show that the Zeno behavior is excluded, we show that there exists a positive lower bound on the inter-event times for all agents. Let $t^{\prime}$ be the latest event time for agent $i$ such that the measurement error is reset 
to zero at time $t^{\prime}$, i.e. $\theta_{i}^{x}\left(t^{\prime}\right)=\theta_{i}^{v}\left(t^{\prime}\right)=0$, which results in $\left.f_{i}\left(t^{\prime}, \theta_{i}^{x}\left(t^{\prime}\right), \theta_{i}^{v}\left(t^{\prime}\right)\right)\right)<0$. For the time between $t^{\prime}$ and the next event time, the following inequality can be obtained from properties of the norm:

$$
\left\|\theta_{i}^{x}(t)\right\| \leq\|\theta(t)\| \leq \int_{t^{\prime}}^{t}\|\dot{\theta}(s)\| d s
$$

Wherein the time-derivative of $\theta$ becomes

$$
\dot{\theta}(t)=\left[\begin{array}{c}
\dot{\theta}^{x}(t) \\
\dot{\theta}^{v}(t)
\end{array}\right]=\left[\begin{array}{c}
v\left(t^{\prime}\right)-\dot{x}(t) \\
-\dot{v}(t)
\end{array}\right]=\left[\begin{array}{c}
\theta^{v}(t) \\
-u(t)
\end{array}\right] .
$$

Taking norm of $\dot{\theta}(t)$ gives

$$
\|\dot{\theta}(t)\| \leq\|\theta(t)\|+\|u(t)\| .
$$

From the MAS model (7), the norm of the control $u(t)$ is

$$
\begin{aligned}
\|u(t)\| & =\left\|\left[\begin{array}{ll}
-\alpha L_{x} & -\beta L_{v}
\end{array}\right]\left(\theta(t)+\left[\begin{array}{l}
x(t) \\
v(t)
\end{array}\right]\right)\right\| \\
& \leq M_{q}(\|\theta(t)\|+\|P\|\|z(t)\|)
\end{aligned}
$$

Consider (8) and (13), the norm of $z(t)$ can be evaluated by

$$
\|z(t)\| \leq e^{\operatorname{Re}\left(\lambda_{3}\right) t}\|z(0)\|+\sum_{j=1}^{n} \frac{\sigma_{j} M_{q}\left\|P^{-1}\right\| e^{-\gamma_{j} t}}{-\left(\operatorname{Re}\left(\lambda_{3}\right)+\gamma_{j}\right)} .
$$

Based on above analysis in (15), (16), (17), (18), the norm in (14) becomes

$$
\begin{aligned}
\left\|\theta_{i}^{x}(t)\right\| & \left.\leq \int_{t^{\prime}}^{t}(1) \| M_{q}\right)\|\theta(s)\|+M_{q}\|P\|\|z(s)\| d s \\
& \leq \tilde{M} \int_{t^{\prime}}^{t} e^{-\underline{\gamma s}} d s \leq \tilde{M} e^{-\underline{\gamma} t^{\prime}}\left(t-t^{\prime}\right),
\end{aligned}
$$

with constant

$$
\widetilde{M}=\hat{\sigma}\left(1+M_{q}\right)+M_{q} M_{p}\left\|\begin{array}{l}
x(0) \\
v(0)
\end{array}\right\|+\frac{\hat{\sigma} M_{p} M_{q}^{2}}{-\left(\operatorname{Re}\left(\lambda_{3}\right)+\bar{\gamma}\right)},
$$

where $\hat{\sigma}=\sum_{j=1}^{n} \sigma_{j}, \quad \underline{\gamma}=\min \left\{\gamma_{j}\right\} \quad$ and $\quad \bar{\gamma}=\max \left\{\gamma_{j}\right\}$ for $j \in\{1, \ldots, n\}$. The next event time $t>t^{\prime}$ will not occur before $f_{i}\left(t, \theta_{i}^{x} t, \theta_{i}^{v}(t)\right)$ crosses zero. As a result, the inter-event time is bounded from below by a positive time $\tau_{D}=t-t^{\prime}$, which is the solution of $\widetilde{M} \tau_{D}=\bar{\sigma} e^{-\underline{\gamma} \tau_{D}}$, where $\bar{\sigma}=\max _{j \in\{1, \ldots, n\}}\left\{\sigma_{j}\right\}$.

\section{Centralized Event-triggered Control}

In some applications, there exists a global master station that collects the information of the whole system and determines the triggering time. In this centralized case, the sequence of event times is denoted by $\left\{t_{k}\right\}_{k \in \mathbb{N}}$, where $t_{k}>t_{k-1} \geq t_{0}$, and it corresponds to a sequence of control updates $\left\{u\left(t_{k}\right)\right\}_{k \in \mathbb{N}}$ with the following control law

$$
u(t)=-\alpha L_{x} x\left(t_{k}\right)-\beta L_{v} v\left(t_{k}\right), \quad t \in\left[t_{k}, t_{k+1}\right) .
$$

Theorem 3.2: Consider the multi-agent systems (1) with the consensus protocol (19) under a centralized eventtriggering schedule and assume that the graphs $\mathcal{G}_{x}$ and $\mathcal{G}_{v}$ are heterogeneously connected. Let the event times $\left\{t_{k}\right\}_{k \in \mathbb{N}}$ be defined by the update rule

$$
f(t, \theta)=\|\theta(t)\|-\sigma e^{-\gamma t}>0
$$

where $\sigma>0,0<\gamma<\operatorname{Re}\left(\lambda_{3}\right)$. Then for any initial conditions $x(0), v(0) \in \mathbb{R}^{n}$, all the agents reach consensus exponentially. Furthermore, the inter-event times $\left\{t_{k+1}-t_{k}\right\}_{k \in \mathbb{N}}$ are lower bounded by a positive constant, i.e., the MAS does not exhibit Zeno behavior.

Proof: Similar to the analysis in Theorem 3.1, a reduced-order model can be derived from (8) as

$$
\dot{\hat{z}}=\tilde{J} \hat{z}+\left[\begin{array}{c}
q_{3}^{\top} \\
\vdots \\
q_{2 n}^{\top}
\end{array}\right] \hat{L} \theta,
$$

where $\hat{z}=\left[z_{3}^{\top} \ldots z_{2 n}^{\top}\right]^{\top}$. By solving above differential equation and taking the norm, we have

$$
\begin{aligned}
\|\hat{z}(t)\| & \leq e^{\operatorname{Re}\left(\lambda_{3}\right) t}\|\hat{z}(0)\|+\int_{t_{0}}^{t} e^{\operatorname{Re}\left(\lambda_{3}\right)(t-s)}\left\|P^{-1}\right\|\|\hat{L} \theta(s)\| d s \\
& \leq e^{\operatorname{Re}\left(\lambda_{3}\right) t}\|\hat{z}(0)\|+\frac{\sigma M_{q}\left\|P^{-1}\right\|}{-\left(\operatorname{Re}\left(\lambda_{3}\right)+\gamma\right)} e^{-\gamma t},
\end{aligned}
$$

which takes $\left\|p_{3}, \ldots, p_{2 n}\right\|^{\top} \leq\left\|P^{-1}\right\|$ into consideration. Since $0<\gamma<\operatorname{Re}\left(\lambda_{3}\right)$, we can verify that $\lim _{t \rightarrow \infty}\left\|z_{i}(t)\right\|=0$ for $i \in\{3, \ldots, 2 n\}$. To exclude the Zeno behavior, the anaylsis parallels what is already presented in the proof of Theorem 3.1. Specifically, let $t^{\prime}$ be the latest event time for MAS and denote $\bar{M}=\sigma\left(1+M_{q}+\frac{M_{p} M_{q}}{-\left(\operatorname{Re}\left(\lambda_{3}\right)+\gamma\right)}\right)+M_{p}\left\|\begin{array}{c}x(0) \\ v(0)\end{array}\right\|$. As a result, the measurement error bounded by

$$
\|\theta\| \leq \bar{M} \int_{t^{\prime}}^{t} e^{-\gamma} t^{\prime} d s \leq \bar{M} e^{-\gamma t^{\prime}}\left(t-t^{\prime}\right),
$$

which implies that there exists a lower bound on the interevent times $t-t^{\prime}=\tau_{D}$, which is implicitly given by the analytical solution of following formula $\bar{M} \tau_{D}=\sigma e^{-\gamma \tau_{D}}$.

\section{NUMERICAL EXAMPLES}

In this section, some simulation examples are presented to demonstrate the effectiveness of the proposed event-triggered control laws in heterogeneous MAS with double-integrator characteristics.

The MAS under investigation consists of five agents; the communication graph $\mathcal{G}_{x}$ is graph 1 and the velocity graph is $\mathcal{G}_{v}$ be graph 2 for the centralized and distributed case. Five agents launch
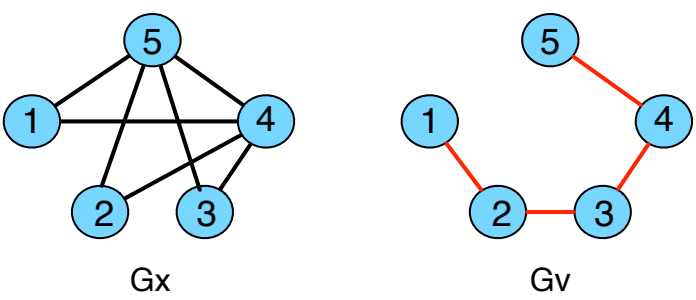

Fig. 1. Communication topologies for numerical example 

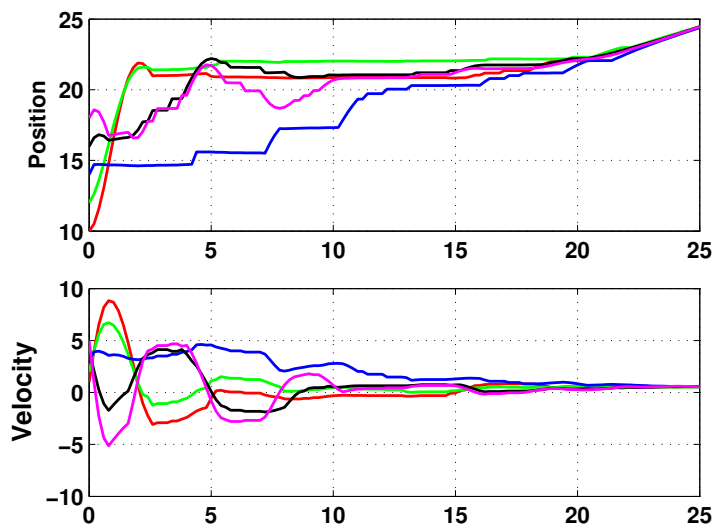

Fig. 2. Consensus under the distributed event-triggered control
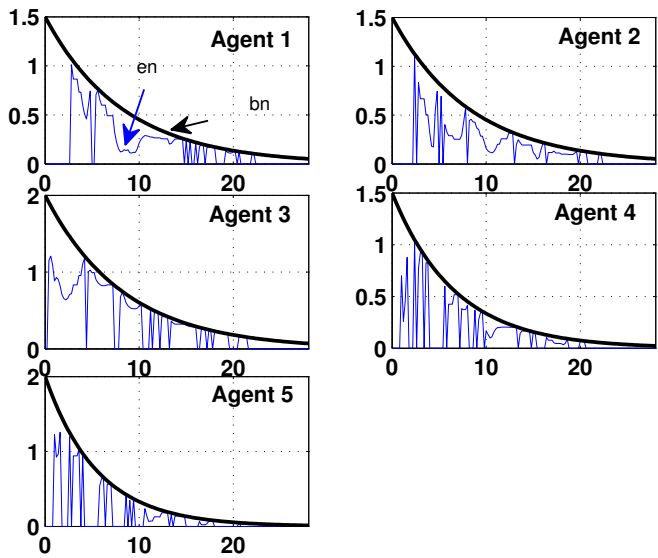

Fig. 3. Evolution of distributed event-triggered function $f_{i}(i=1, \ldots, 5)$

from initial position $\quad x(0)=[10,12,14,16,18]^{\top}$ and velocity $v(0)=[1,2,3,4,5]^{\top}$. In distributed casse, set $\alpha=1, \beta=1.5, \quad \sigma_{i}=[1.5,1.5,2,1.5,2]$ and $\gamma_{i}=[0.12,0.12,0.12,0.15,0.2]$ for $i \in\{1, \ldots, 5\}$. As shown in the Figure 2, the MAS achieves the position and velocity consensus. Moreover, the corresponding event-triggered function for each agent can be found in Figure 3, where the event-triggered rates of agents are in turn $(30 \%, 15 \%, 16 \%, 32 \%, 47 \%)$.

In the centralized case, $\sigma=2.5$ and $\gamma=0.1$ are chosen. Figure 4 shows the MAS under centralized eventtriggered control achieves position and velocity consensus asymptotically. Figure 5 shows the the norm of measurement error in the centralized case stays below the specified timedependent threshold.

\section{CONCLUSIONS}

Second-order consensus problems of MASs under eventtriggered update rules are investigated in this paper. The agents with double integrator dynamics exchange information over a heterogenous network where the information topologies of position and velocity are different in the most general case. In addition, the convergence of the consensus protocol based on event-triggered scheduling is examined in,
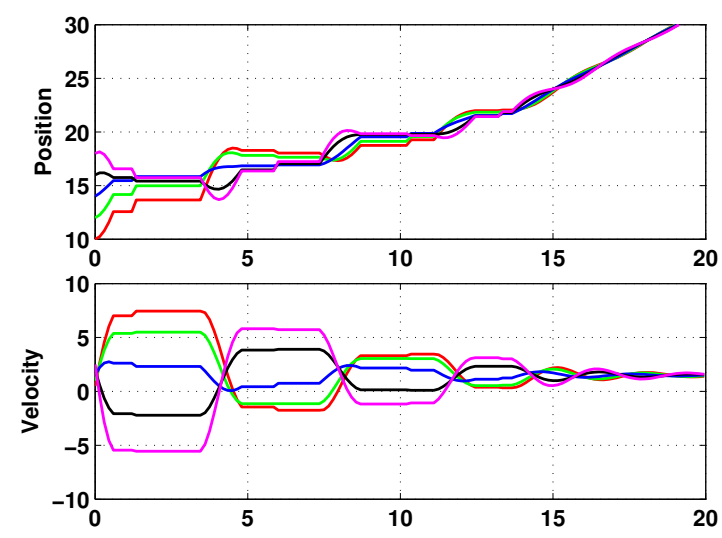

Fig. 4. Consensus under centralized event-triggered control

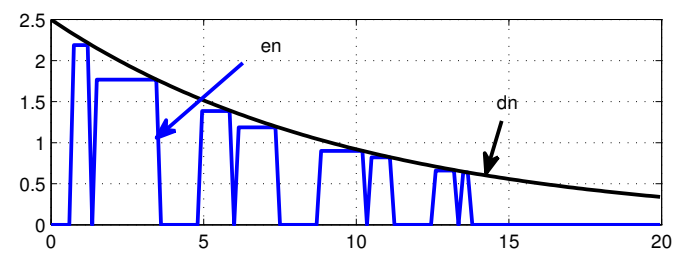

Fig. 5. Evolution of centralized event-triggered function $f$

the distributed and centralized case. The proposed eventtriggering strategies not only preserve the desired convergence properties, but also guarantee exclusion of the Zeno behavior in both cases. Future work involves the investigation of transmission delay, and packet loss case for event-based control in second-order consensus problem.

\section{REFERENCES}

[1] R. Olfati-Saber and R. Murray, "Consensus problems in networks of agent with switching topology and time-delays," IEEE Transactions on Automatic Control, vol. 49, pp. 1520-1533, 2004.

[2] W. Ren and E. Atkins, "Distributed multi-vehicle coordinated control vi local information exchange," International Journal of Robust and Nonlinear Control, vol. 17, pp. 1002-1033, 2007.

[3] D. Goldin and J. Raisch, "Consensus for agent with double integrator dynamics in heterogenous networks," Asian Journal of Control, vol. 15 , pp. 1-10, 2013.

[4] K. Aström and B. Bernhardsson, "Comparison of riemann and lebesgue sampling for first order stochastic systems," (USA), pp. 2011-2016, in Proc. 41st IEEE Conf. Decision and Control, 2002.

[5] P. Tabuada, "Event-triggered real-time scheduling of stabilizing control tasks," IEEE Transactions on Automatic Control, vol. 54, pp. 452-467, 2007.

[6] X. Wang and M. Lemmon, "Self-triggered feedback control systems with finite-gain $\mathcal{L}_{2}$ stability," IEEE Transactions on Automatic Control, vol. 54, pp. 452-467, 2009.

[7] D. Dimarogonas, E. Frazzoli, and K. Johansson, "Distributed eventtriggered control multi-agent systems," IEEE Transactions on Automatic Control, vol. 57, pp. 1291-1297, 2012.

[8] D. Kingston, W. Ren, and R. Beard, "Consensus algorithms are inputto-state stable," (USA), pp. 1686-1690, in Proc. Amer. Cont. Conf., 2005

[9] G. Seyboth, D. Dimarogonas, and K. Johansson, "Event-based broadcasting for multi-agent average consensus," Automatica, vol. 49, pp. 245-252, 2013.

[10] C. Godsil and G. Royle, Algebraic Grpah Theory. Springer-Verlag Graduate Texts in Mathematics New York, 2001.

[11] I. Gohberg, P. Lancaster, and L. Rodman, Matrix Polynomials. Classics in Applied Mathematics, 2009. 